\title{
Evaluación comparativa de redes KBANN y sistemas ANFIS para la localización de fallas en redes de distribución de energía eléctrica
}

\author{
Juan J. Mora ${ }^{\S}$ Lucas P. Pérez, Sandra M. Pérez \\ Grupo de Investigación en Calidad de Energía Eléctrica y Estabilidad, \\ Programa de Ingeniería Eléctrica, Universidad Tecnológica de Pereira, Colombia \\ §e-mail:jjmora@utp.edu.co
}

(Recibido: Septiembre 27 de 2007 - Aceptado: Noviembre 20 de 2008)

\begin{abstract}
Resumen
La regulación actual sobre calidad de la energía eléctrica enfatiza los requerimientos relacionados con la continuidad del servicio, aumentando así la importancia del problema de localización de fallas en redes de distribución de energía eléctrica. La oportuna localización de fallas acelera el procedimiento de restauración del suministro en redes de distribución. En este artículo se presenta una evaluación comparativa de dos enfoques para la localización de fallas. Estos enfoques combinan la lógica difusa, las redes neuronales y las reglas de experto, y utilizan registros de las componentes de frecuencia fundamental de tensión y de corriente medidas en la subestación de energía. Las pruebas realizadas en una red de distribución de energía muestran errores promedio inferiores al 5\% en la localización de fallas, validando los enfoques propuestos en este trabajo.
\end{abstract}

Palabras clave: Energía eléctrica, Localización de fallas, Redes de distribución, Redes KBANN, Sistemas ANFIS.

ELECTRICAL ENGINEERING

\section{Comparative evaluation of KBANN networks and ANFIS systems for fault location in electrical power distribution networks}

Current regulation on electrical power quality emphasizes the requirements related to service continuity, thereby increasing the importance of the fault location problem in electrical power distribution networks. The opportune location of faults accelerates the procedure for distribution network supply restoration. This paper presents an evaluative comparison of two approaches to solve the fault location problem. These approaches combine fuzzy logic with artificial neural networks and expert's rules, and use records of the fundamental frecuency components of current and voltage measured at the power substation. Tests carried out in a power distribution network show average errors lower than $5 \%$ in fault location, validating the approaches proposed in this work.

Keywords: Electrical energy, Fault location, Distribution networks, KBANN networks, ANFIS systems. 


\section{Introducción}

Un tema de gran actualidad e importancia en el sector eléctrico es la calidad de la energía, ya que en la mayor parte del mundo los nuevos esquemas de regulación exigen que las empresas de energía presten un mejor servicio. Para el caso colombiano, la Comisión de Regulación de Energía y Gas (CREG), y la Superintendencia de Servicios Públicos Domiciliarios (SSPD), han reglamentado los índices asociados a la duración y frecuencia de las interrupciones mediante el establecimiento del índice DES (duración equivalente de las interrupciones), y el índice FES (frecuencia equivalente de las interrupciones), para cada tipo de circuito (CREG, 2002-2005).

Una de las principales causas de la pérdida de continuidad en los sistemas eléctricos se encuentra en las fallas paralelas, el $80 \%$ de las cuales ocurren en el sistema de distribución, según diferentes estudios (Das, 1998). Por esto, la localización oportuna de fallas en sistemas de distribución permite el mejoramiento de los índices DES y FES. El restablecimiento depende del tiempo de la localización de la falla, que en la mayoría de empresas distribuidoras y debido a problemas de infraestructura, está asociado a las posibles llamadas telefónicas de usuarios afectados o al tiempo que demora la cuadrilla en localizar la falla mediante una inspección visual de toda la red (Das, 1998; Mora, 2006).

La localización de fallas en sistemas de distribución es compleja debido a que éstos presentan un alto número de laterales. Las metodologías basadas en el modelo eléctrico de la red proporcionan información sobre la distancia asociada a la impedancia estimada desde la subestación hasta el lugar donde ha ocurrido la falla (Takagi et al., 1981; Novosel et al., 1998; Choi et al., 2004). Debido al gran número de laterales, la distancia estimada se cumple para varios laterales del sistema, convirtiendo éste en un problema de múltiple estimación que complica la ubicación del lateral bajo la condición de falla (Mora, 2006).

Como alternativa de solución al problema de localización de fallas en sistemas de distribución, en este trabajo se propone una metodología que usa diferentes características extraídas de señales de tensión y de corriente medidas en la subestación de distribución. Las características extraídas, denominadas descriptores, se usan para entrenar dos técnicas de inteligencia artificial basadas en la capacidad de aprendizaje de las redes neuronales artificiales y en el conocimiento experto contenido dentro de la lógica difusa, combinándolas dentro de dos metodologías probadas en los problemas de localización de fallas, como son las redes KBANN (Pérez et al, 2007) y los sistemas ANFIS (Mora et al, 2006).

En la Sección 2 se presentan los fundamentos más importantes de las técnicas empleadas. En la Sección 3 se muestra la manera de adquirir las características o descriptores empleados para la etapa de entrenamiento, a partir de las señales de tensión y corriente medidas en la subestación y cuando el sistema se encuentra bajo condición de falla y prefalla. En la Sección 4 se presenta en forma detallada, la metodología propuesta para realizar la localización de la falla. En la Sección 5 se muestra una serie de resultados comparativos de ambas técnicas, cuando se realizan pruebas de localización de fallas usando un sistema de prueba prototipo. En la Sección 6 se presentan las conclusiones más importantes de esta investigación.

\section{Fundamentos de las técnicas de clasificación}

Las técnicas de clasificación basadas en inteligencia artificial han incrementado su popularidad en la solución tanto de tareas de clasificación como de regresión en la estimación de valores futuros de funciones o tendencias de datos. La superioridad de una técnica frente a otra radica en la estructura algorítmica y matemática que usen para resolver el problema de minimización del error.

A continuación, se presentan los fundamentos de dos técnicas de clasificación que usan la lógica difusa y las redes neuronales para resolver problemas de clasificación, y que se aplican en este artículo a la localización de fallas en sistemas de distribución. 


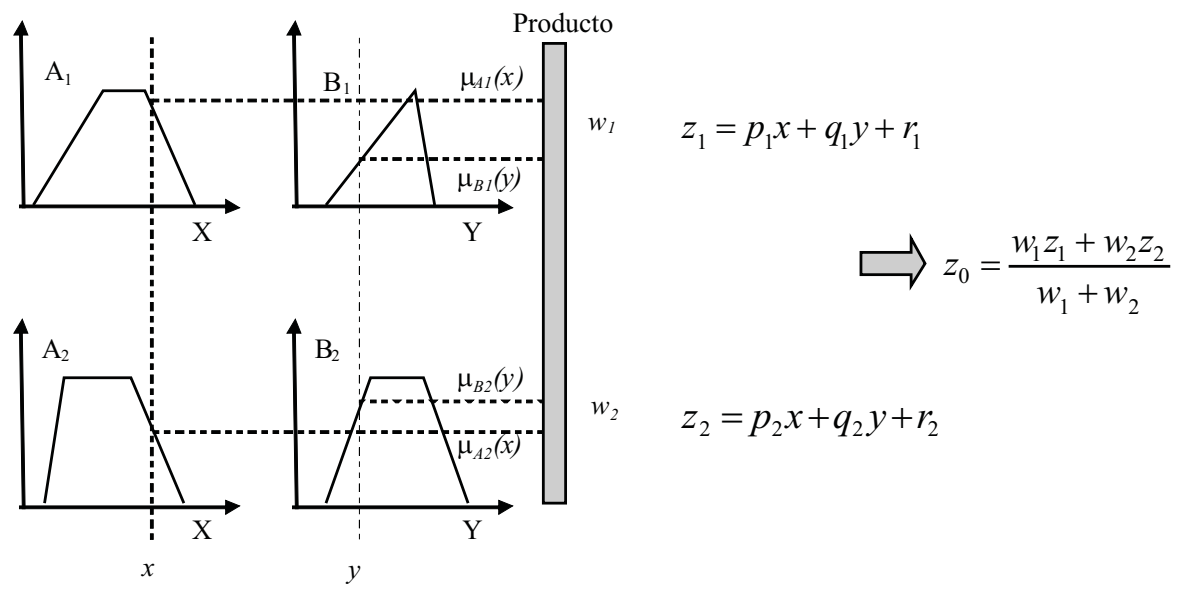

Figura 1. Sistema de inferencia Takagi-Sugeno de primer orden.

\subsection{Sistema de inferencia adaptivo neurodifuso (ANFIS)}

Esta técnica combina el conocimiento experto (plasmado en reglas difusas) con la capacidad de aprendizaje que poseen las redes neuronales artificiales (Mora et al, 2006).

El modelo ANFIS consiste en un sistema híbrido neurodifuso, el mismo que es funcionalmente equivalente al mecanismo de inferencia TakagiSugeno (T-S) (Rusell \& Norvig, 2002). Para un sistema de inferencia T-S de primer orden, un conjunto de reglas difusas IF-THEN se presentan en (1) y (2), donde $A_{i}$ y $B_{i}$ son conjuntos difusos. La Figura 1 presenta una sencilla representación de este mecanismo de inferencia.

Regla 1:

Si $x$ es $A_{1}$ y $y$ es $B_{1}$, entonces $z_{1}=p_{1} x+q_{1} y+r_{1}$

Regla 2:

Si $x$ es $A_{2}$ y $y$ es $B_{2}$, entonces $z_{2}=p_{2} x+q_{2} y+r_{2}$

Los niveles de activación de las reglas se calculan como $w_{1}=A_{i}(x), B_{i}(x), i=1,2, \ldots$, donde el operador lógico AND puede modelarse por una $t$-norma continua como el producto. Las salidas individuales de cada regla se obtienen como una combinación lineal de los parámetros del antecedente de cada regla, así: $z_{i}=p_{i} x+q_{i} y+r_{i}$, $i=1,2, \ldots$
La salida de control $z_{0}$ del modelo se obtiene por la normalización de los grados de activación de las reglas, y está dada por la salida individual de cada regla, así:

$$
z_{0}=\frac{w_{1} z_{1}+w_{2} z_{2}}{w_{1}+w_{2}} \Rightarrow z_{0}=\bar{w}_{1} z_{1}+\bar{w}_{2} z_{2}
$$

donde $\bar{w}_{1} \mathrm{y} \bar{w}_{2}$ son los valores normalizados de $w_{1}$ $y w_{2}$ con respecto a la suma $\left(w_{1}+w_{2}\right)$. La red neuronal híbrida que representa este tipo de inferencia es una red adaptable con 5 capas, donde cada capa representa una operación del mecanismo de inferencia difusa tal como se representa en la Figura 2. En esta arquitectura, todos los nodos de una misma capa tienen la misma función (los representados por cuadros son nodos adaptables, es decir que sus parámetros $p, q$ y $r$ son ajustables). La estructura de la red ANFIS consta de 5 capas (Mora et al, 2006), así:

Capa 1. Las entradas de esta capa corresponden a $x$ y $y$. La salida del nodo es el grado de pertenencia para el que la variable de entrada satisface el término lingüístico asociado a este nodo.

Capa 2. Cada nodo calcula el grado de activación de su regla asociada. Ambos nodos están representados con una $T$ en la Figura 6, debido que pueden representar cualquier $t$-norma para modelar la operación lógica AND. Los nodos de esta capa son conocidos como nodos de reglas. 


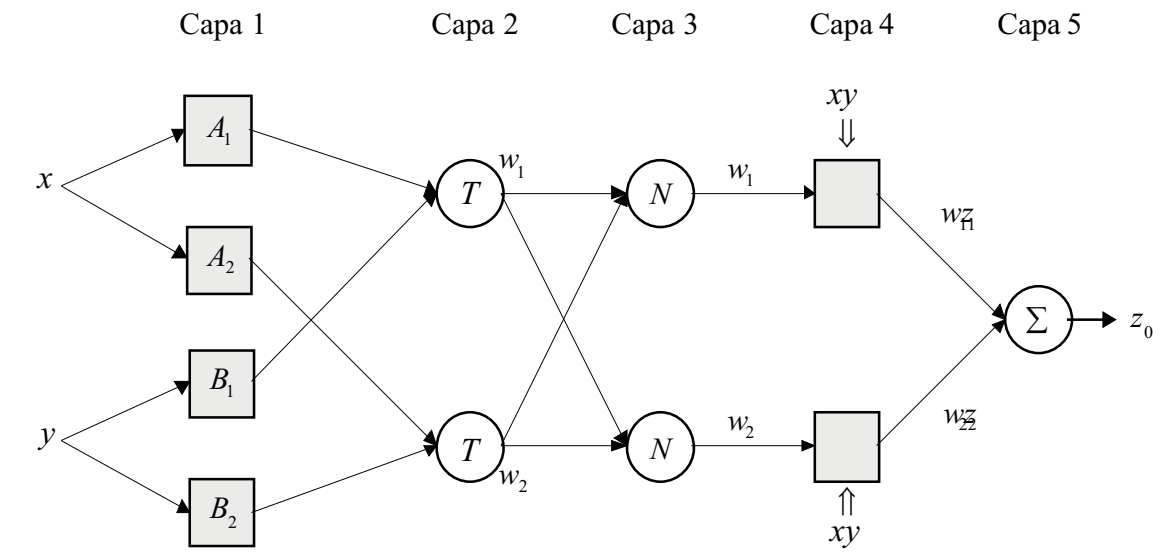

Figura 2. Arquitectura ANFIS equivalente para inferencia Takagi-Sugeno de primer orden.

Capa 3. Cada nodo en esta capa está representado por $N$ en la Figura 2, para indicar la normalización de los grados de activación. La salida del nodo es el grado de activación normalizado de la regla $i$, con respecto a la suma de los grados de activación.

Capa 4. La salida de los nodos corresponde al producto del grado de activación normalizado por la salida individual de cada regla.

Capa 5. El único nodo de esta capa calcula la salida total del sistema (agregación), como la suma de sus entradas individuales.

A partir de este sencillo procedimiento, se crea una red tipo ANFIS entrenada para localizar fallas.

\subsection{Redes neuronales artificiales basadas en el conocimiento (KBANN)}

Estas redes neuronales son entrenadas usando un conjunto de entrenamiento conformado por reglas IF-THEN y empleando un razonamiento de tipo Mandani (Rusell and Norvig, 2002).

El punto de partida de este sistema es la denominada teoría de dominio. Se trata de sistemas inductivos que utilizan un conjunto de reglas aproximadamente correctas, inferidas de un dominio o problema específico, para intentar describir lo que se conoce sobre dicho dominio.
El algoritmo KBANN traduce cada regla de partida que describe el comportamiento de un sistema en su equivalente en forma de red neuronal artificial, ofreciendo una primera aproximación a la arquitectura y pesos de las conexiones entre los distintos elementos de proceso. Posteriormente, se aplica el algoritmo de retro-propagación del error para reajustar la red y refinar su comportamiento (Towell, 1991).

Al aplicar el algoritmo KBANN, las reglas se transforman en redes neuronales en las que los pesos de los enlaces correspondientes a antecedentes no negados se les asigna un valor $+\omega$, las conexiones con antecedentes negados se les asigna pesos con valor - $\omega$, y el término de polarización del elemento de proceso que genera el consecuente toma un valor $-(2 P-1) \omega / 2$, donde $P$ es el número de premisas no negadas. Para codificar una regla disyuntiva, KBANN asigna el valor $\omega$ a los pesos de cada enlace con los antecedentes, mientras que al término de polarización del elemento de proceso que genera el consecuente se le asigna el valor $-\omega / 2$. En la Figura 3 se muestran las subestructuras generadas por las reglas.

El algoritmo KBANN traduce una colección de reglas en una red neuronal, tomando cada regla de forma individual y transformándola en una subred que reproduzca de manera precisa el comportamiento de dicha regla. 


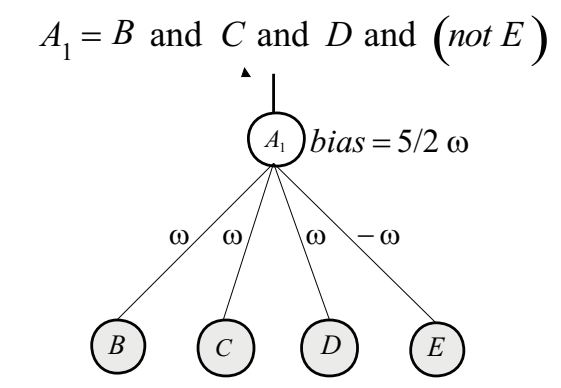

(a)

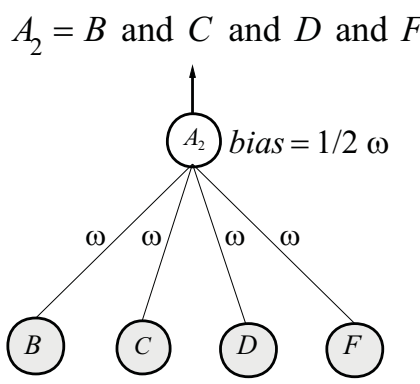

(b)

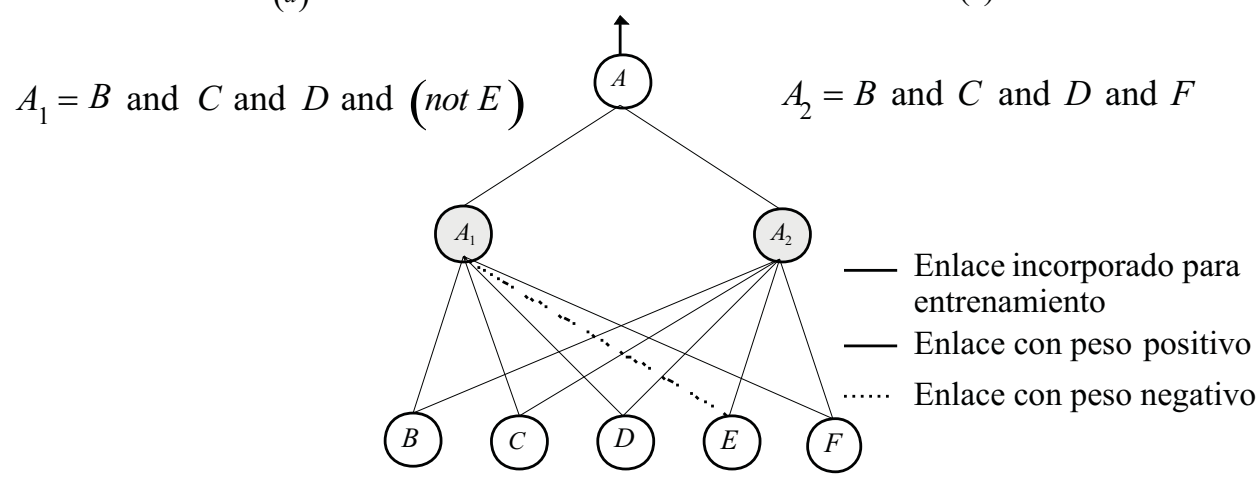

(c)

Figura 3. Conversión de un conjunto de reglas a una red neuronal artificial.

Todas las subredes se ensamblan en una red neuronal cuyo comportamiento debe representar el de la regla completa.

Una vez obtenida la red completa tal como se presenta en la Figura 3c, ésta se completa con aquellas conexiones que no se incluyen de forma explicita a través de las reglas, con un valor de peso aleatorio. A partir de aquí, y por medio del algoritmo de retropropagación del error y los datos de entrenamiento, se ajusta el sistema para que el resultado final sea una refinación del original (Pérez et al., 2007).

\section{Caracterización del sistema}

\subsection{Particularidades de los sistemas de distribución de energía eléctrica}

Los sistemas de distribución presentan una gran complejidad para la realización de diversos estudios. Para el caso de localización de fallas, algunos problemas se deben principalmente a que estos sistemas no son balanceados, tienen una topología radial con muchas ramificaciones o laterales monofásicos y cargas intermedias. Adicionalmente, las líneas no son homogéneas debido a que normalmente las redes se expanden con diferentes calibres de conductores y a medida que los usuarios así lo requieren (Mora, 2006).

En los sistemas de distribución de energía eléctrica, debido a la gran cantidad de subestaciones y la baja potencia que alimenta cada circuito, se cuenta con pocos elementos de protección y automatización. En el mejor de los casos, los sistemas de distribución tienen medidores de tensión de barra y corriente total del transformador $\mathrm{y}$ algunas protecciones como relevadores de sobrecorriente (función ANSI 50/51), en la subestación. En algunos casos, se cuenta también con elementos propios de automatización de los sistemas de distribución como reconectadores, seccionalizadores y fusibles ubicados a lo largo del sistema (Horowitz \& Padke, 1995; Blackburn, 1998). 


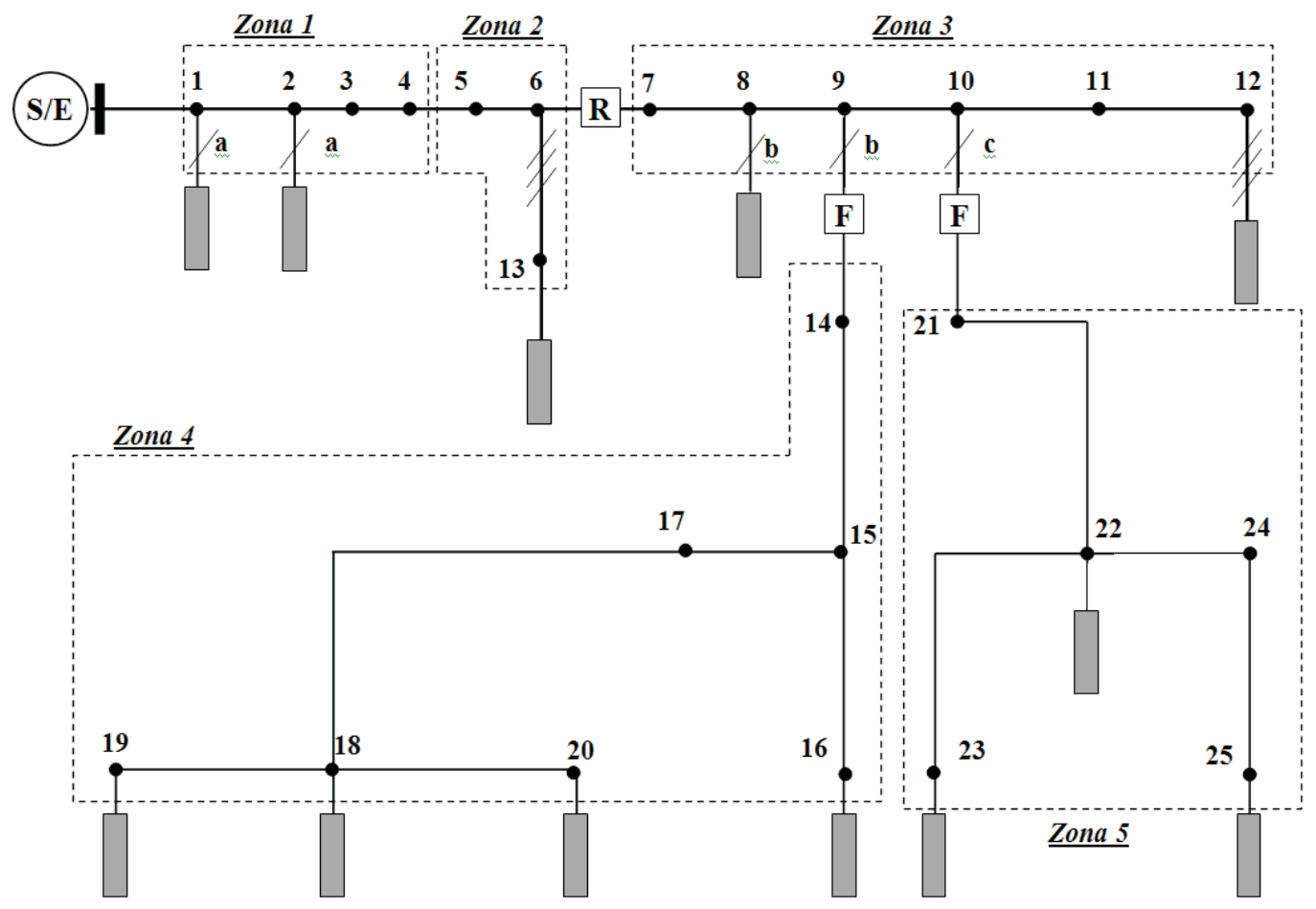

Figura 4. Sistema de distribución típico.

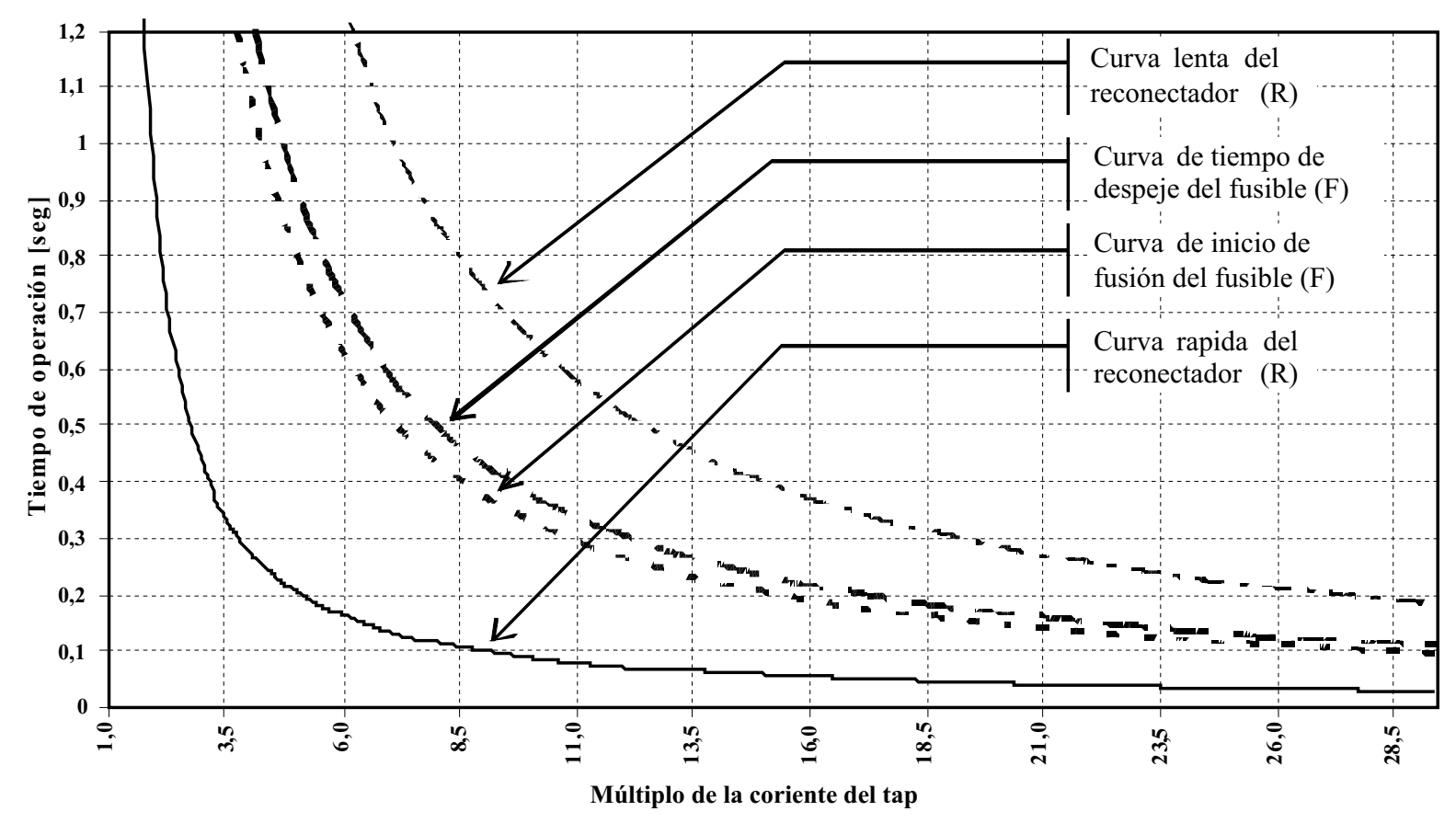

Figura 5. Curvas de relación inversa tiempo-corriente. 


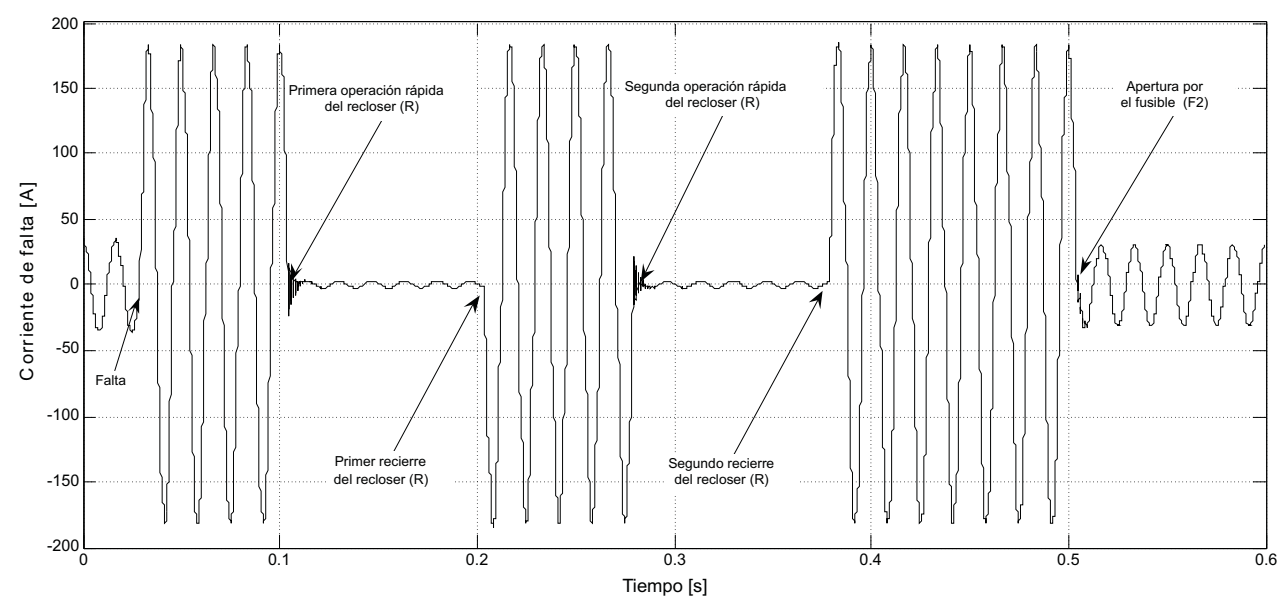

Figura 6. Registro de corriente ante la ocurrencia de una falla de fase a tierra permanente en el nodo 22 del sistema de la Figura 4.

Para el circuito de distribución utilizado para las pruebas de la metodología propuesta y que se muestra en la Figura 4, un reconectador automático $(\mathrm{R})$ está configurado para abrir y cerrar nuevamente el circuito ante fallas aguas abajo del elemento, de forma coordinada con los fusibles (F).

La secuencia puede contener uno o más disparos rápidos y recierres para despejar fallas transitorias y restaurar el servicio. En caso de que la falla persista, los fusibles retiran todas aquellas que ocurren más allá de su ubicación (Pérez et al., 2007). Este tipo de coordinación es conocida como salvamento de fusibles, y un esquema genérico de las curvas de coordinación con relés de relación inversa tiempo-corriente se presentan en la Figura 5.

\subsection{Información importante para el problema de localización de fallas}

Aunque la mayor parte de la información que describe al sistema de distribución bajo estado de falla, consiste básicamente en los registros en el dominio del tiempo de tensión y corriente medidos en el nodo de la subestación, existe información adicional que es útil para resolver el problema de localización de fallas. El registro de llamadas telefónicas realizadas por los clientes informando sobre fallas en la red, la tipificación de la carga (tipo de carga que existe en cada nodo industrial comercial y residencial, y para éste último, la estratificación social) y las condiciones climáticas, son ejemplos de este tipo de información.

Esta información que permite caracterizar el sistema bajo falla constituye los llamados descriptores y aquellos utilizados en esta investigación se describen a continuación.

\subsubsection{Vector del tiempo de conmutación del circuito $(t)$}

En este vector se almacenan los tiempos obtenidos de los registros de corriente resultantes de la conmutación de los elementos de protección del sistema de distribución. La actuación de reconectadores, seccionalizadores, interruptores en conjunto con relés de sobrecorriente instantáneos y de relación inversa tiempo corriente y fusibles, depende de su tiempo de calibración (Blackburn, 1998; Lee et al., 2004). La Figura 6 muestra las señales de corriente medidas en el nodo de la subestación cuando se ha presentado una falla permanente fase a tierra en el nodo 22 del circuito de la Figura 4. La configuración de las protecciones está dispuesta en el esquema de salvamento de fusibles tal como 
se muestra en la Figura 5, de modo que ante una falla corriente abajo de un fusible, actúa primero el reconectador respectivo y finalmente el fusible. Por medio de la lectura de los tiempos de operación de los elementos de protección se puede asociar una zona de falla, determinada por la localización en el circuito del elemento de protección respectivo.

\subsubsection{Variación de la corriente de carga (I)}

Cuando se presenta una falla en el sistema, ésta es clarificada por alguno de los elementos de protección, lo cual implica la desconexión de usuarios y la consecuente disminución de la corriente de carga. Dependiendo de la cantidad de carga desconectada, se puede estimar cuál de los dispositivos de protección actuó. En la Figura 6 se aprecia que durante la acción rápida del reconectador es desconectada gran parte de la carga en la fase fallada, pero finalmente, cuando el fusible interrumpe la falla, la carga desconectada no es tan importante. El descriptor de variación de la corriente está conformado por un vector de tres elementos, uno para cada fase.

\subsubsection{Variación de la potencia aparente $(\Delta S)$}

La disminución de carga ocasionada por la operación de las protecciones, ocasiona una diferencia entre la potencia antes y después de la falla. Conociendo el valor de la carga que fue desconectada, se puede inferir la ubicación de la falla dentro del sistema de distribución. El descriptor de variación de la potencia aparente está conformado por un vector de tres elementos, uno para cada fase.

\subsubsection{Variación de la tensión y del factor de $\operatorname{potencia}\left(\Delta V, f_{p}\right)$}

Asociados a la salida de carga por la acción de las protecciones, ocurren cambios en la tensión y el factor de potencia, entre los valores de prefalla y posfalla. Estos cambios también se usan como descriptores o información asociada al lugar de ocurrencia de la falla. Los descriptores de variación de la tensión $\Delta V \mathrm{y}$ del factor de potencia $f_{p}$ están conformados por un vector de tres elementos, uno para cada fase en cada uno de los casos.

\section{Metodología de localización propuesta}

La metodología propuesta consiste en entrenar el sistema ANFIS y las redes KBANN, con una base de datos que contiene los descriptores anteriormente propuestos y obtenidos de simulaciones en un sistema de prueba. En el entrenamiento se analizan varias alternativas de configuración de los localizadores a partir de la variación de las entradas, para lo cual considerando 5 posibles entradas $(t, \Delta I, \Delta S, \Delta V$, $\Delta f_{p}$ ) el número de combinaciones probables es igual a $2^{5}-1=31$.

El sistema de distribución se debe dividir en zonas. Cada zona se determina de tal forma que la localización de una de ellas no implique múltiple estimación del sitio de falla, tal como se presenta en el ejemplo de aplicación en la Sección 5.

\subsection{Entrenamiento}

Para este caso se proponen varios conjuntos de descriptores como datos de entrada. Como dato de salida se tiene la zona en la cual se simula la falla. La metodología se fundamenta en la generación de relaciones entre entradas y salidas que permitan localizar las fallas ante nuevas entradas de descriptores.

\subsection{Generación de reglas}

La generación de reglas se realiza para introducir el conocimiento teórico propio de la identificación previa de la zona de falla para los dos sistemas, generadas a partir de la determinación de las variaciones obtenidas del vector $\boldsymbol{t}$. Usando los descriptores es posible conocer la zona donde la falla ocurrió e introducir esta información en una regla tipo Takagi-Sugeno para el sistema ANFIS o una regla tipo Mandani para la red KBANN. Los antecedentes de las reglas corresponden a los valores de los descriptores usados en esta investigación para cada una de las zonas en las cuales se divide el sistema de distribución y los consecuentes son las zonas en las que se dividió el sistema. 


\subsection{Proceso de validación}

El proceso de validación consiste en probar los 31 diferentes localizadores de fallas entrenados previamente, usando datos que no fueron usados en la fase de entrenamiento. El error en la estimación de la zona de falla se calcula así:

$$
\text { Error }[\%]=\frac{\begin{array}{l}
\text { Datos de } \\
\text { validación }
\end{array}-\begin{array}{l}
\text { Datosbien } \\
\text { clasificados }
\end{array}}{\begin{array}{l}
\text { Datos de } \\
\text { validación }
\end{array}} \times 100
$$

\section{Pruebas y resultados}

\subsection{Sistema de prueba}

El sistema de distribución de $25 \mathrm{kV}$ de la empresa SaskPower \& Light en la ciudad de Saskatoon en Canadá, es utilizado como prototipo de prueba en este articulo (Das, 1998). El circuito fue simulado usando el software para estudio de fenómenos transitorios Alternative Transients Program (ATP), y su diagrama unifilar se presenta en la Figura 4.

\subsection{División del sistema por zonas}

Para tener las etiquetas a identificar por los sistemas ANFIS y KBANN, el sistema se divide por zonas, de tal manera que cuando los métodos localicen la región del sistema de potencia donde ocurrió la falla, no haya problema de múltiple estimación. Según lo anterior, sólo hay un circuito o ramal por zona para que la cuadrilla de mantenimiento realice la búsqueda y reparación de la falla.

La división por zonas propuesta para el circuito de prueba corresponde a la presentada en la Figura 4, en la cual se muestra que el circuito de prueba se divide en 5 zonas diferentes a ser identificadas como zonas en falla.

Para tener suficientes medidas del sistema de distribución, se simularon múltiples tipos de fallas en diferentes localizaciones. Los tipos de fallas que se simularon usando el software ATP en el sistema de distribución de la Figura 4 son: falla monofásica a tierra, falla bifásica, falla bifásica a tierra y falla trifásica.

Tabla 1. Resultados obtenidos en la localización de la falla, para los mejores 15 localizadores.

\begin{tabular}{ccc}
\hline Combinación de & \multicolumn{2}{c}{ Porcentaje de error en la localización de la falla } \\
\cline { 2 - 3 } descriptores de entrada & ANFIS & KBANN \\
$\Delta \mathbf{V}$ & 12.8 & 13.4 \\
$\Delta \mathbf{I}$ & 10.6 & 4.7 \\
$\Delta \mathbf{S}$ & 7.4 & 8.6 \\
$\Delta \mathbf{f}_{\mathrm{p}}$ & 9.2 & 11.8 \\
$\mathbf{t}$ & 0.8 & 7.5 \\
$\Delta \mathbf{I}, \mathbf{t}$ & 8.3 & 10.5 \\
$\Delta \mathbf{S}, \Delta \mathbf{I}$ & 8.7 & 9.3 \\
$\Delta \mathbf{I}, \Delta \mathbf{V}$ & 5.1 & 8.4 \\
$\Delta \mathbf{S}, \mathbf{t}$ & 7.2 & 6.2 \\
$\Delta \mathbf{I}, \Delta \mathbf{f}_{\mathbf{p}}$ & 6.3 & 6.1 \\
$\Delta \mathbf{I}, \Delta \mathbf{S}, \mathbf{t}$ & 9.5 & 10.7 \\
$\Delta \mathbf{\mathbf { f } _ { \mathrm { p } }}, \mathbf{t}, \Delta \mathbf{V}$ & 13.6 & 12.1 \\
$\Delta \mathbf{S}, \Delta \mathbf{I}, \Delta \mathbf{S}$ & 5.1 & 9.1 \\
$\Delta \mathbf{V}, \Delta \mathbf{I}, \Delta \mathbf{S}, \mathbf{t}$ & 4.7 & 8.4 \\
$\Delta \mathbf{V}, \Delta \mathbf{I}, \Delta \mathbf{S}, \mathbf{t}, \Delta \mathbf{f}_{\mathrm{p}}$ & 6.2 & 7.5 \\
\hline
\end{tabular}


Estas fallas fueron simuladas en cada uno de los nodos del sistema, considerando que existen ramales monofásicos en los cuales sólo se puede simular un solo tipo de falla. Además, cada una de las fallas se simuló con seis resistencias diferentes $(0.5 \Omega, 5 \Omega, 10 \Omega, 20 \Omega, 30 \Omega$ y $40 \Omega)$, para conformar una base de datos de 2985 fallas.

\subsection{Estructura de las pruebas}

Las pruebas realizadas con el fin de validar las dos metodologías propuestas consisten en evaluar las 31 combinaciones posibles de localizadores, usando los datos que no fueron empleados en la etapa de entrenamiento. Del total de registros de falla se utilizaron 1005 registros de entrenamiento y los 1985 restantes como registros de prueba.

Las 31 combinaciones posibles de descriptores definen el mismo número de localizadores basados en ANFIS o en KBANN y se realizan para determinar las relaciones entre aquellos que permitan obtener menores errores en la localización de fallas.

En la Tabla 1 se presentan los 15 mejores resultados obtenidos en la localización de la falla para las dos metodologías y aquellos conjuntos de descriptores que mejor desempeño muestran.

\subsection{Análisis de resultados}

A partir de la Tabla 1, que contiene los resultados de las pruebas, se puede notar la coincidencia de las mejores 15 alternativas de localización a partir de la definición de las entradas. Las restantes combinaciones no muestran mejores resultados a los presentados en la Tabla 1.

Adicionalmente, y para el caso del sistema de prueba, se puede notar que utilizando el localizador basado en redes ANFIS, el mejor resultado se obtuvo usando el descriptor de los tiempos de conmutación $(\boldsymbol{t})$ para la corriente de carga, debido que este descriptor contiene información topológica del sistema y se obtiene del tiempo de calibración de los reconectadores del sistema de distribución. Para KBANN, el descriptor de variación de corriente $(I)$, presenta los mejores resultados en cuanto a la localización de la zona en falla.

Cuando se usan combinaciones de descriptores, se encuentran resultados de error similares entre metodologías, aunque el error promedio para el método de localización es de $7.7 \%$, mientras que para el KBANN es de $9.0 \%$. De la prueba extensiva de los 31 localizadores conformados con cada una de las metodologías y a partir de la Tabla 1, se muestran las alternativas para seleccionar diferentes descriptores, lo cual brinda diversas posibilidades para la implementación en un sistema real.

\subsection{Perfeccionamiento de los localizadores}

A partir de los análisis de los resultados mostrados en la Tabla 1, se puede apreciar que la mayoría de

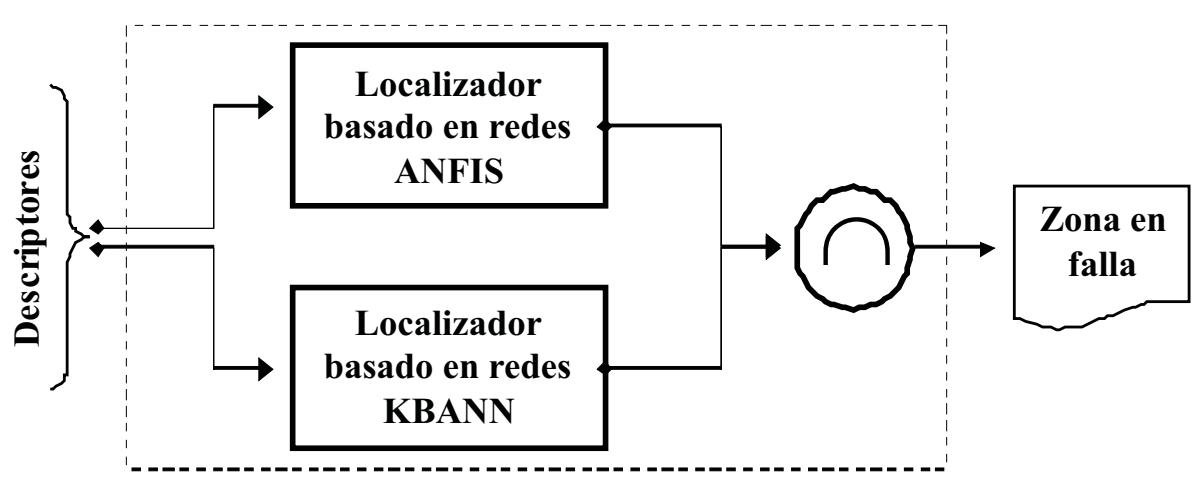

Figura 7. Arquitectura del localizador perfeccionado que usa una estrategia cooperativa entre redes ANFIS y KBANN. 


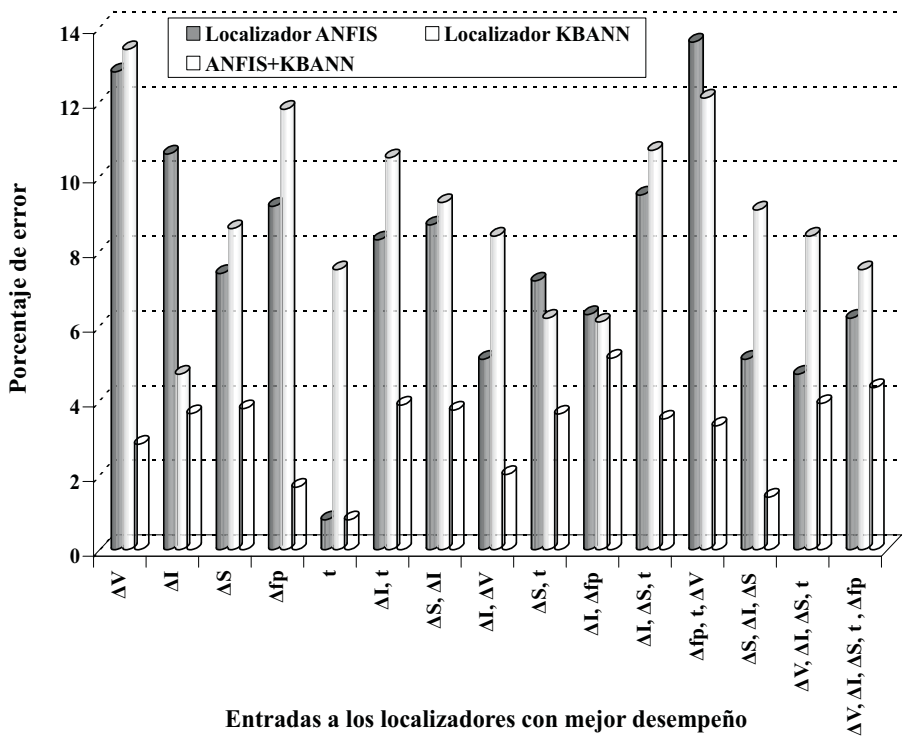

Figura 8. Resultados comparativos del error en la localización de fallas de los localizadores basados en redes ANFIS y KBANN.

los errores ocurren para fallas localizadas en nodos que se encuentran en el límite de la definición de la zona. Este hecho se puede solucionar si se utilizan dos alternativas de zonificación complementarias y se asume como la respuesta más probable aquella que contenga la intersección de las dos respuestas entregadas por los localizadores.

La estrategia de perfeccionamiento incluye el reconocimiento de la zona usando redes ANFIS como primer localizador, según la zonificación propuesta en la Figura 4. Un segundo localizador basado en redes KBANN se utiliza con una zonificación con cuatro zonas, definidas y entrenadas utilizando los datos de los nodos limitantes de cada una de las zonas presentadas en la Figura 4. El resultado final se obtiene mediante la intersección de las zonas obtenidas por cada localizador, tal como se presenta en la Figura 7.

En la Figura 8 se muestran gráficamente los resultados comparativos del error en la localización para las redes ANFIS y KBANN trabajando de forma individual, así como el localizador perfeccionado que se presenta en esta sección.

\section{Conclusiones}

En este artículo se realiza la comparación entre los sistemas ANFIS y las redes KBANN para solucionar el problema de localización de fallas en sistemas de distribución, usando las características extraídas de las señales de tensión y corriente medidas en la subestación.

El sistema de prueba modelado con ATP, corresponde a un sistema de distribución real con una alta complejidad. Los resultados obtenidos con ambos enfoques permiten validarlos como solución al problema, ya que los errores promedio individuales de los casos presentados son inferiores al $10 \%$ en la localización de la falla. Con la estrategia de perfeccionamiento, el error se reduce a un promedio de $2.6 \%$. La localización del sitio donde ocurrió la falla ayuda a mejorar los índices de continuidad del servicio (DES y FES), mejorando así la calidad de la potencia.

\section{Referencias bibliográficas}

Blackburn, J. L. (1998). Protective relaying: principles and applications. Second Edition, Marcel Dekker Inc. 
Choi, M.-S., Lee, S.-J., Jin, B.-G., \& Lee, D.-S. (2004). A new fault location algorithm using direct circuit analysis for distribution systems. IEEE Transactions on Power Delivery 19 (1), $35-41$.

CREG (Comisión de Regulación de Energía y Gas). (2002-2005). Resolución CREG 084 de 2002, CREG 113 de 2004, CREG 024 de 2005.

Das R. (1998). Determining the locations of faults in distribution systems. Doctoral Thesis, Department of Electrical Engineering, University of Saskatchewan, Saskatoon, Canada.

Horowitz, S., \& Phadke, A. (1995). Power system relaying. Second Edition, Research Studies Press.

Lee, S.-J., Choi, M.-S., Kang, S.-H., Lin, B.-G., Lee, D.-S., Ahn, B.-S., Yoon, N.-S., Kim, H.-Y., \& Wee, S.-B. (2004). An intelligent and efficient fault location and diagnosis scheme for radial distribution systems. IEEE Transactions on Power Delivery 19 (2), 524-532.

Mora, J. J. (2006). Localización de fallas en sistemas de distribución. Tesis Doctoral, Universidad de Girona, España.

Mora-Flórez, J. J., Pérez-Hernández, L. P., \& Pérez-Londoño, S. M. (2006). Utilización de redes ANFIS y señales de corriente para la localización de falla en sistemas de distribución de energía eléctrica. Ingeniería e Investigación 26(3), 93-99.
Novosel, D., Hart, D., Hu, Y., \& Myllymaky, J. (1998). System for locating faults and estimating fault resistance in distribution networks with tapped loads. United States Patent No. 5,839,093.

Pérez, L. P., Pérez, S. M., \& Mora, J. J. (2007). Análisis de las redes KBANN para localización de fallas en sistemas de distribución. Simposio Internacional de la Calidad de la Energía Eléctrica, Manizales, Colombia.

Rusell, S. J., \& Norvig, P. (2002). Artificial intelligence: a modern approach. Second Edition, Prentice Hall.

Takagi, T., Yamakoshi, Y., Baba, J., Uemura, K., \& Sakaguchi, T. (1981). A new algorithm of an accurate fault location for EHV/UHV transmission lines: Part I-Fourier transformation method. IEEE Transactions on Power Apparatus and Systems PAS-100 (3), 1316-1323.

Towell, G. (1991). Symbolic knowledge and neural networks; insertion, refinement and extraction. Doctoral Thesis, Department of Computer Sciences, University of Wisconsin, Madison, U.S.A. 